\title{
CARACTERÍSTICAS, NOTAS DISTINTIVAS Y TENDENCIAS DE LA GLOBALIZACIÓN: EL PRÓXIMO ESCENARIO GLOBAL
}

\section{FEATURES, DISTINCTIVE ASPECTS AND TRENDS OF GLOBALIZATION: THE NEXT GLOBAL STAGE}

\author{
Adrián Alejandro Flores Konja * \\ Docente Principal de la Facultad de Ciencias Contables -UNMSM \\ Manuel Hidalgo Tupia** \\ Docente de la Facultad de Ciencias Contables - UNMSM
}

[Recepción: Febrero de 2011/ Conformidad: Abril de 2011]

\section{RESUMEN}

El fenómeno de la globalización, complejo y cambiante, ha impactado grandemente en todos los ámbitos, indudablemente, pero su comprensión conceptual se hace difícil, por sus propias características. Entre los esfuerzos más saltantes y reconocidos de su enfoque conceptual resalta el de Kenichi Ohmae, quien ha sintetizado las características, notas distintivas y tendencias de la globalización en sus aspectos político y económico. En este informe preliminar de una investigación exploratoria se describe las características y tendencias señaladas por Ohmae, y se las compara con las observaciones de los investigadores sobre el ámbito global y la realidad nacional, proyectando en esta realidad las tendencias presentadas por Ohmae.

Señala que los principales protagonistas en la escena global han cambiado; con la desaparición o "reinvención" de los Estados nacionales, y la aparición de los "Estados región”; también se ha reducido la intervención del Estado en las decisiones económicas, siendo también esta una nota distintiva de la globalización. En lo económico, señala las tres características más importantes de la

\begin{abstract}
The phenomenon of globalization, complex and changing, has greatly impacted in all areas, no doubt, but conceptual understanding is made difficult by its very nature. Among the most outstanding efforts and recognized its conceptual approach highlights the Kenichi Ohmae, who has summarized the characteristics, distinctive characteristics and trends of globalization on political and economic aspects. In this preliminary report describes an exploratory investigation of patterns and trends identified by Ohmae, and compared with the observations of researchers on the global and national reality in this reality by projecting the trends presented by Ohmae.

Ohmae Indicates that the main protagonists in the global scene have changed, with the disappearance or "reinvention" of national states, and the emergence "States region." also reduced state intervention in economic decisions, this also being a hallmark of globalization in economics, said the three most important features of the global economy, which are to be boundless, to be invisible and earnings multiply.
\end{abstract}

* Doctor en Ciencias Contables y Empresariales- UNMSM; Contador Público Colegiado Certificado. Actual Decano de La Facultad de Ciencias Contables, UNMSM. E- mail: afloreskonja@yahoo.es

** Magister en Ciencias Económicas- UNMSM; Docente de la Facultad de Ciencias Contables- UNMSM.

E- mail: mhidalgot@hotmail.com 
economía global, que son no tener fronteras, ser invisible y manejarse con ganancias que se multiplican.

Según Ohmae, las plataformas de la globalización, son: la lengua inglesa, el sistema operativo Windows, el dólar norteamericano y las marcas, $y$, en segundo lugar, la nueva cultura empresarial, las redes de cajeros electrónicos, y las transacciones electrónicas. Enfatiza en el "cibercontinente", como el nuevo "lugar" de la economía, desplazando en importancia a la economía física.

Las tendencias formuladas por Ohmae en lo económico el 2005 han sido negadas por la realidad de las crisis financiera norteamericana de 2008, y su reto al sistema del dólar, y los factores que Ohmae deja de lado, como la geopolítica y el narcotráfico, pero también la irrupción de los estados nacionales de pueblos- continente como China, India y Rusia están tomando fuerza, en algunos casos a favor y en otros contrapuestos a las tendencias esbozadas.

Palabras claves: Economía Global, Escenario Global, Estado-Región, Estado-Nación, Globalización, Apertura Económica. Dólar. Reserva Federal. Sociedad de la Información, Escenario, Dirección, Guión, Estrategia.

\section{INTRODUCCION}

El presente es un informe preliminar de una investigación exploratoria sobre las características y tendencias de la globalización y su impacto en nuestro país, el cual se enfocó en los aportes de Kenichi Ohmae, en su obra "El próximo escenario global. Retos y Oportunidades en nuestro mundo sin fronteras", que es, precisamente, un intento de plantear las principales características y tendencias de la globalización, presentadas por dicho autor como de una necesidad indetenible e inmutable.

El objetivo de esta investigación exploratoria fue identificar las características, notas distintivas y tendencias del mundo globali-

\begin{abstract}
According to Ohmae, the platforms of globalization are: the English language, the Windows operating system, the U.S. dollar and trademarks, and, secondly, the new corporate culture, ATM networks and electronic transactions. Emphasis on the "cibercontinente" as the new "place" of the economy, instead physical economy.

Made by trends in economic Ohmae 2005 have been denied by the reality of the American financial crisis of 2008, and its challenge to the dollar system, and factors Ohmae aside, such as geopolitics and drug trafficking, but also the emergence of national statescontinent nations such as China, India and Russia are gaining strength in some cases for and others against the grain of the trends identified.
\end{abstract}

Keywords: Global Economy, Global Scenario, State-Region, Nation-State, Globalization, Economic Opening. Dollar. Federal Reserve. Information Society, Stage, directing, writing, Strategy.

zado en los ámbitos político y económico tal y como lo enfoca Kenichi Ohmae, y analizar los pronósticos presentados en 2005 por dicho autor y determinar las implicancias de estas tendencias y de las políticas globales inspiradas en este enfoque para nuestro país.

Nuestro enfoque metodológico consistirá en describir las características y tendencias, de la globalización, tal y como las plantea Ohmae en forma general, y compararlas con las observadas por el investigador en la realidad peruana. Buscaremos interpretar los hechos $y$ las formulaciones buscando su verdadera esencia, y trataremos de encontrar la coherencia de los hechos dentro de un sistema económico y político global, utilizando para ello el enfoque hermenéutico - cualitativo. 

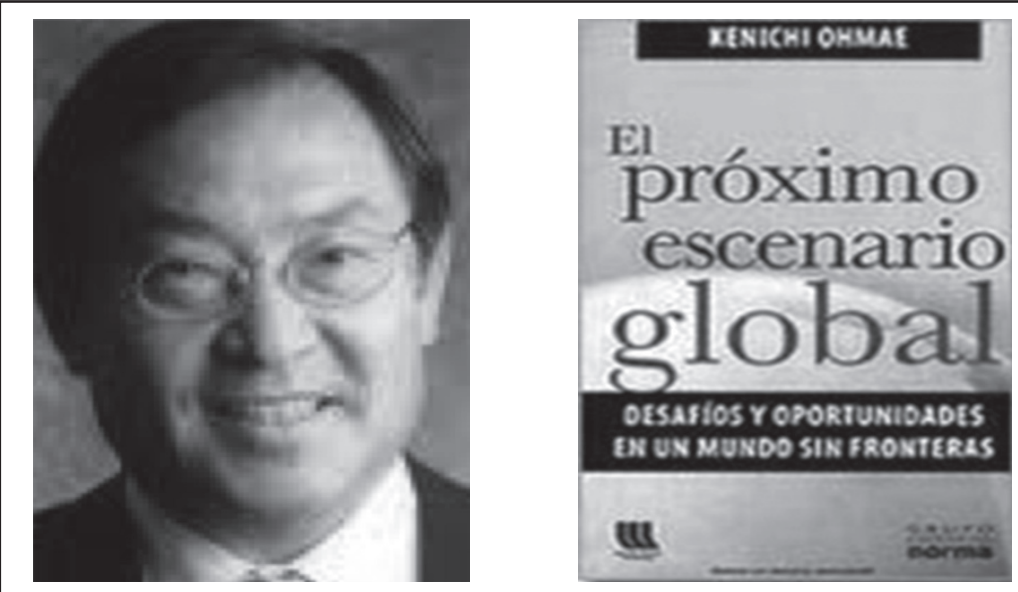

Kenichi Ohmae economista japonés, nacido en la isla Kyushi en 1943.

Cabe notar que el análisis del libro, de 2005, no alcanza los hechos de la crisis financiera internacional de septiembre de 2008 y sus repercursiones, lo que nos permitirá comparar mejor en el tiempo las formulaciones.

\section{Características y notas distintivas de la Globalización}

En la obra mencionada, Ohmae señala tres grandes partes: el escenario, es decir, el contexto global en lo político y económico; la dirección escénica, como la estructura de poder en la toma de decisiones globales, y el guión, o las políticas esbozadas a aplicarse por la dirección escénica. Siendo que sobre el escenario y el guion abundaremos en lo siguiente, mencionaremos que Ohmae menciona dentro de la dirección escénica a los países de la Triada: Estados Unidos, Unión Europea y Japón, sin identificar dentro de estas sociedades los agentes en que reside el poder, tanto económico como político, dentro del esquema empresarial y financiero.

Ohmae es un economista japonés, nacido en la isla Kyushi en el año 1943, tiene un largo historial en empresas occidentales, especialmente McKinsey \& Company, y en conocidos centros académicos, como UCLA y Wharton School. En las últimas 3 décadas, Ohmae se convirtió en uno de los principales estrategas mundiales en el terreno empresarial, un gurú sobre estrategia empresarial,. Escribe para los principales voceros del eje financiero City de Londres - Wall Street, como son The Wall Street Journal, The New York Times y Harvard Business Review; con el respaldo de Wharton School, uno de los "think tanks" más influyentes del núcleo financiero de la Triada y que auspició el libro de la referencia, el más beneficiado con la globalización, para proponer un esquema que fundamente $y$ - entendemos --- ayude a perpetuar la forma actual de la globalización, en línea con Fukuyama, Brzesinski y Toffler, entre otros. Los títulos de sus libros son elocuentes de ello, en especial, "El Mundo sin Fronteras" y "El fin del Estado nación".

Según el resumen autorizado publicado por Leader Summaries, Ohmae "realiza predicciones con altas probabilidades de cumplirse"; y esto es algo que vamos a analizar.

Según el análisis de Ohmae, la globalización está signada en lo económico por el fin de las fronteras económicas y, en lo político por la desaparición o "reinvención" de los Estados nacionales, y la aparición de los "Estados región", como los nuevos grandes 
protagonistas, abiertos al mercado externo, como es el caso de los modelos que pregona: Singapur (ciudad estado asiático), Irlanda y las regiones chinas del Delta del Rio Perla, o de Liaodong (Delian). Enfoca la crisis de los Estados nacionales desde la perspectiva económica y política, analizando como impacta en este paradigma las realidades de la globalización. Aquí, Ohmae está en línea con las formulaciones de Francis Fukuyama sobre el "fin de la Historia" que representaría la globalización, con su postulación del "fin del estado nacional", arguyendo que la economía globalizada será un agente efectivo para este fin del estado. Ohmae se dirige a los "estados - región para cooptarlos en la globalización perpetua con la promesa del protagonismo y el bienestar. ¿Tiene bases para que la promesa no se convierta en ilusión?

\section{La economía global y el fin de la econo- mía tradicional}

Esta tiene tres características más importantes, que son según Ohmae, no tener fronteras, ser invisible y manejarse con ganancias que se multiplican.

En el primer caso, las fronteras de los estados nacionales pierden importancia, em- pezando por la apertura comercial o reducción general de los aranceles, y en general de los controles estatales. Las fuerzas económicas que colaboran con la desaparición de las fronteras son las comunicaciones, el capital, las corporaciones y los consumidores, que se ven cada vez mas como realidades transnacionales.

El carácter de invisible se debe a que las transacciones ylos pagos están deslocalizados, puesto que se hacen a través de las computadoras, como por ejemplo las transacciones B2B (Business to business), B2C (Business to consumer) y C2C (Consumer to consumer), cuyos pagos se realizan mediante tarjetas de crédito. Otra realidad conexa es la existencia de redes mundiales de cajeros automáticos, que permiten hacer efectivo un débito bancario en cualquier parte del mundo.

Por su parte, la existencia de un exceso de capital de los ciudadanos de los países industrializados hace que puedan invertirse en otras regiones del mundo, incrementando su valor, de tal manera que estos recursos pueden llegar a cualquier región del mundo que sepa atraerlos. Aquí se hace referencia al altísimo crecimiento de la Inversión Extranjera Directa de los años recientes.
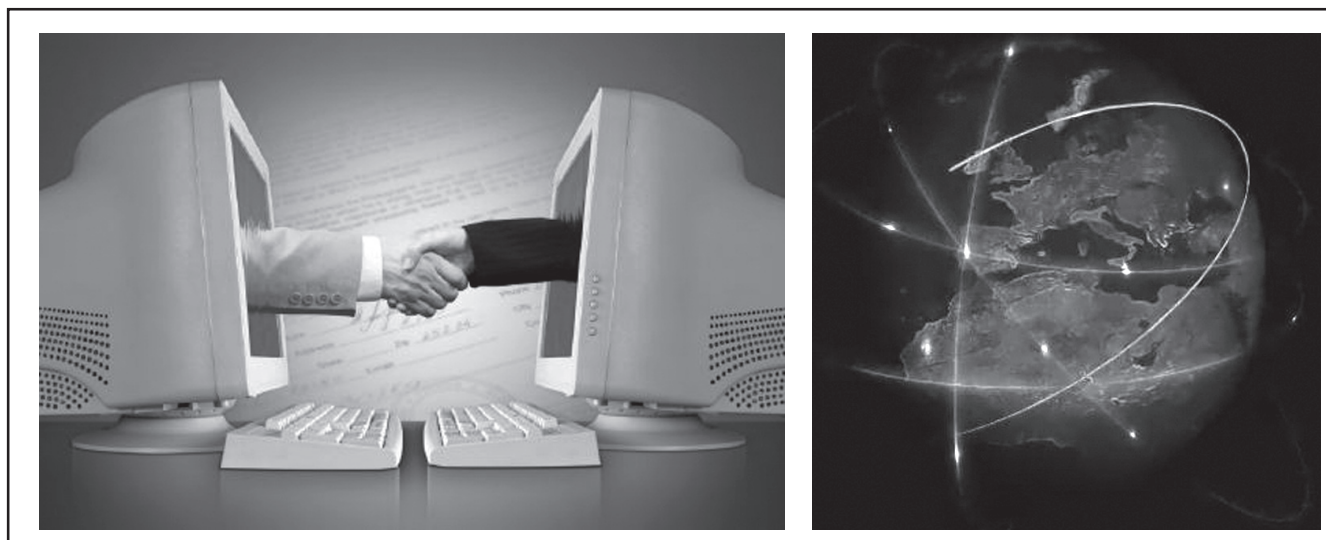

Las realidades del comercio electrónico han transformado la forma de hacer negocios, permitiendo una perspectiva de internacionalización para las empresas. 
Cuadro No 1. Las nuevas realidades económicas internacionales

En los últimos 25 años:

- El comercio mundial ha crecido al doble que la producción mundial.

- La inversión extranjera directa (I.E.D.) tres veces mas rápido.

- La negociación transfronteriza en activos financieros diez veces más veloz.

Este escenario global se origina en 1985, según Ohmae, cuando se plantan las semillas del mismo que son la creación del sistema operativo Windows y el fracaso de los Acuerdos Plaza del Grupo de los 5 Países Mas Desarrollados de ese año, cuyo objetivo era proteger al viejo estilo a la mayor economía física del mundo, la estadounidense, del cambio de paradigmas. Otra de las semillas fue la “apertura” de China, en los 90.

Según Ohmae, también se ha acabado el elemento de la intervención del Estado en las decisiones económicas, siendo también esta una nota distintiva de la globalización. Ohmae afirma que las plataformas de la globalización, son: la lengua inglesa, el sistema operativo Windows, el dólar norteamericano y las marcas, y, en lugar algo secundario, la nueva cultura empresarial, las redes de cajero electrónicos, y las transacciones electrónicas usando las tarjetas de crédito.

Respecto al dólar, Ohmae señala la importancia que para el esquema tiene la política del entonces presidente de la Reserva Federal de los EEUU, Alan Greenspan, de convertir al dólar en la moneda de atesoramiento mundial, tanto de las personas como de los gobiernos, atrayendo la inversión del mundo, y obteniendo las ventajas de un endeudamiento sin limites para los Estados Unidos, con el triple déficit de deuda, comercial y presupuestario, como sucede en la actualidad.
Ohmae habla mucho sobre el cibercontinente, que es más grande - en número de transacciones -que Europa, donde "habitan" los "ciberitas", poniendo una gran esperanza en este desarrollo, es decir, en el comercio electrónico. Por ejemplo, alguien digita lo que le interesa en un buscador, y encuentra a la empresa que lo posee; hace el pago electrónicamente mediante una tarjeta de crédito, y como tercer paso, la empresa, mediante un sistema de logística internacional, DHL, por ejemplo, le hace llegar el bien. Ohmae señala que el tercer paso, logístico, es el que tiene más dificultades.

\section{Los Estados - región son el nuevo prota- gonista}

Ohmae considera que los Estados - nación caerán en una serie de crisis, como los de Europa y los de África, tanto políticas como económicas, y serán reemplazados por los estados - región, como son los casos de Singapur (que es una ciudad estado, en realidad), Irlanda, Finlandia, Delian y muchos otros en China. En Sudamérica, sólo menciona a Sao Paulo, Brasil, pero podemos vislumbrar que algunas regiones peruanas de la costa, como Lima - Callao o La Libertad, potencialmente podrían considerarse como candidatas a "estados región” según este modelo.

\section{Gráfico No 1}
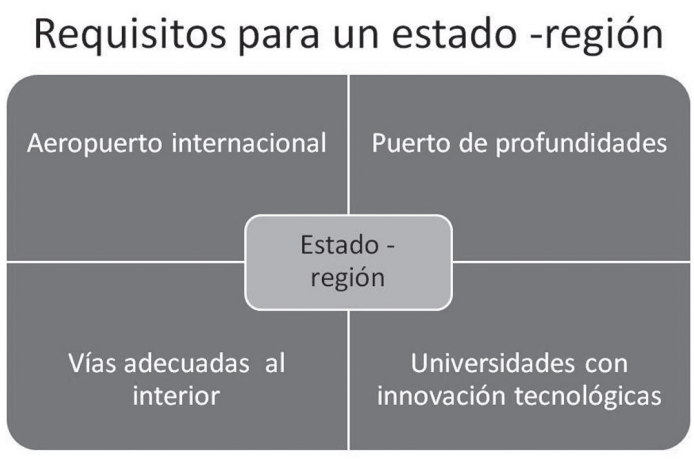

Fuente: Leader Summaries. Op. Cit. Elaboración Propia 


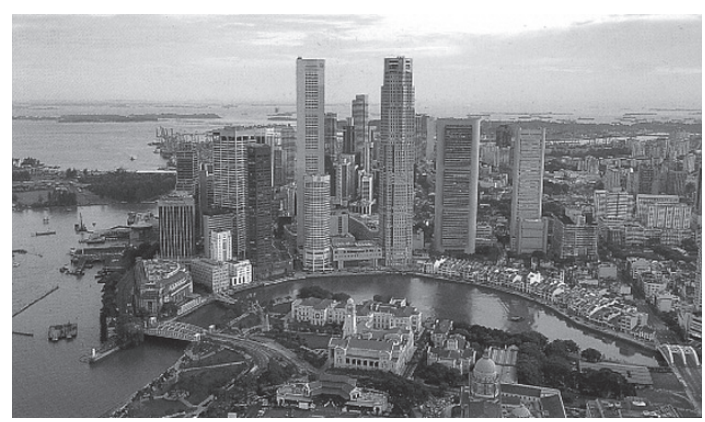

Singapur, ciudad estado de 4 millones y medio de habitantes y $700 \mathrm{~km} 2$, que Ohmae considera como un prototipo de "estado región" Sólo el departamento de Lima tiene más de $34,000 \mathrm{~km} 2$, es decir, 50 veces más y cuenta con casi 10 millones de habitantes.

Los principales requisitos de estos Estados - región serían: poseer un aeropuerto internacional, poseer un gran puerto que atienda adecuadamente al comercio internacional, poseer vías de comunicación adecuadas hacia el interior (para la extracción de los recursos), y poseer universidades y centros de estudio superiores de vanguardia, que permitan la rápida incorporación tecnológica.

\section{Los Estados - región y la economía en el Perú}

Las políticas que refleja Ohmae respecto a "refundar" el Estado indudablemente han influido en el diseño de la última reforma del Estado peruano, durante la gran crisis de los 90 y del 2000. Impactado por el Consenso de Washington, el Perú redujo su aparato estatal, las redes de controles y regulaciones, abrió la economía a la inversión extranjera, desrreguló la banca, y por último, procedió a la regionalización. El contexto que ayudo a desmantelar el estado nacional fueron: la restricción de créditos del FMI y el Sistema financiero internacional, a partir de la crisis de la deuda de 1972; la crisis política con la subversión y la presencia del narcotráfico, con su aporte masivo de narcodólares y el financiamiento de sectores completos de la economía informal, como el contrabando y el lavado de dinero.

Los conceptos que presenta Ohmae han estado presentes en especial en la gran reforma peruana que fue la regionalización, proceso de creación de regiones que ha significado un gran cambio, pero que no ha cumplido su promesa de bienestar y empoderamiento para los departamentos afectados por el centralismo. Esto se viene intentando remediar con la creación de las macrorregiones.

Con la apertura de las dos últimas décadas, el ingreso masivo de inversión extranjera directa, y adicionalmente, con la firma del Tratado de Libre Comercio con los Estados Unidos, el Perú ha tenido un incremento notable en el PBI, volcándose gran parte de la economía a la exportación, en especial, en los sectores minero y agrícola, y algunos sectores de manufacturas textiles. Se han prefigurado regiones costeras como Lima Callao, La Libertad, Arequipa, Lambayeque y Piura, con un gran potencial económico, al estilo de los Estados región de Ohmae.

Como se puede apreciar en el mapa adjunto, estas regiones se ubican en la costa, siendo las regiones de la sierra y la selva las convidadas de piedra del banquete del "boom de exportaciones".

\section{Gráfico No 2}

Exportaciones: Regiones más beneficiadas

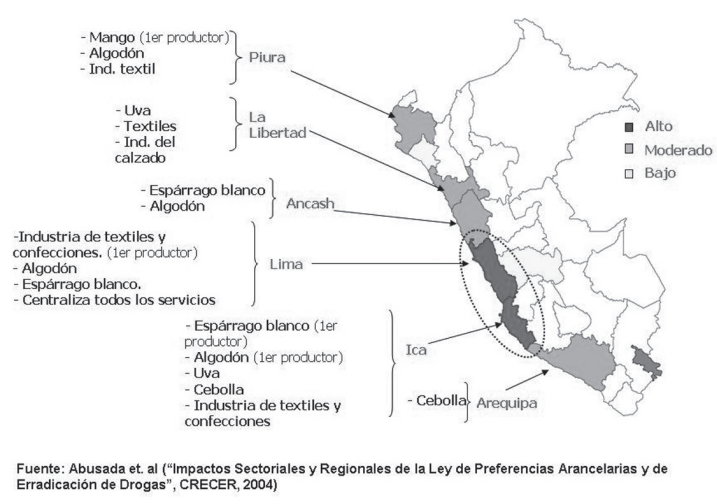

Boom de la exportación: Regiones incluidas ... y excluidas 
Pese al crecimiento de las exportaciones, pocos consideran que este modelo puede extenderse por mucho tiempo. Incluso, desde el punto de vista de los Estados -región de Ohmae, nuestras regiones tienen un déficit mayúsculo en infraestructura y educación. Son elocuentes las preocupaciones expresadas por el gerente de Área de Banca Corporativa del BCP, Luis Alfonso Carrera Sarmiento, en el seminario "Estrategias ante la Fluctuaciones de la Economía Global”, quien expreso que "existen serios retos para la sostenibilidad del crecimiento del país." Agrego que "se proyecta que en los próximos cinco años se invertirán unos US\$ 41.000 millones, pero en este momento no tenemos ni la mano de obra calificada necesaria ni la capacidad de generación de energía suficiente ni la infraestructura requerida para sostenerlas .....) y es difícil que se puedan obtener las licencias sociales requeridas si un tercio de nuestra población sigue viviendo en la pobreza." Los pocos potenciales "estados - región” peruanos tienen pues el gran problema de la falta de sistemas educativos adecuados, y retrasos gravísimos en la infraestructura.

El pasado 13 de octubre de 2010 estuvo en Lima el propio politólogo norteamericano Francis Fukuyama y, a despecho de su fé en la globalización, según el diario "El Comercio" "destacó que el modelo de crecimiento económico que ha aplicado el Perú, basado en la exportación de materias primas, es insostenible." 3 Fukuyama afirmó que el Perú "ha tenido mucha suerte como exportador de commodities porque en los últimos años han tenido mucha demanda."4 Según el periódico limeño "predijo que si hubiera un freno económico, sobre todo en China, los precios caerían y afectarían nuestra economía."5

La situación se complica con el agravamiento de la pobreza en algunas regiones, el aumento de la violencia y de la actividad del narcotráfico, siendo el Perú el primer productor de cocaína del mundo. El Perú se dá el lujo de ser el país de la moneda mas "dura" de la subregión, debido a que el ingreso masivo de narcodólares hace que el BCRP deba salir a comprar los dólares para evitar una apreciación aun mayor del sol peruano, incrementando así sus reservas.

En estas circunstancias, Perú observa el espectro del narcotráfico socavando rápidamente el Estado nacional, en medio de una crisis de seguridad espantosa; y el espectro de un separatismo agresivo como un futuro desastroso en un país multicultural y multiétnico. Es decir, los nuevos Estados -región peruanos no serían capaces de enfrentar la crisis de seguridad causada por las regiones inviables dominadas por el narcotráfico y la violencia; más que "protagonistas", serían las víctimas de un guión geopolítico.

\section{Contrastando los postulados de Ohmae después de la crisis financiera internacio- nal}

Los sucesos de septiembre de 2008, la mayor crisis financiera internacional en la historia, un cataclismo de dimensiones nunca antes vistas, desmienten muchas de las proyecciones de Ohmae, en especial, en su esperanza puesta en la fortaleza - que el nunca pone en tela de juicio - del dólar norteamericano, y la capacidad de ese país de mantener el orden monetario, financiero y comercial

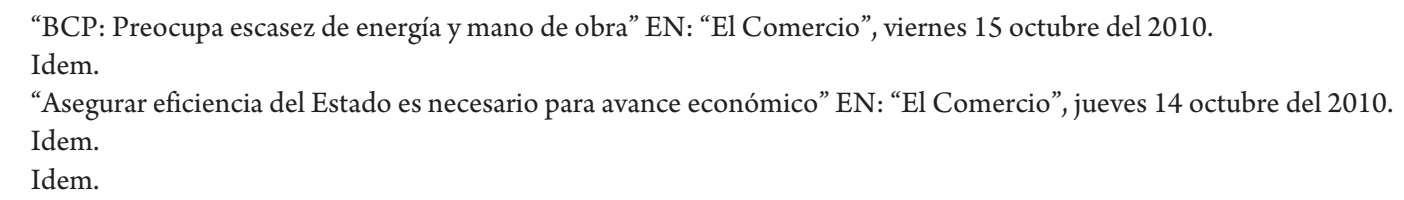


internacional. Resulta anecdótico que sean las políticas de Greenspan, que tanto alaba Ohmae, las señaladas hoy como la causa de la mayor crisis financiera internacional en la historia. La vuelta rápida a la intervención estatal - "rescate bancario" o "nacionalización de la banca" --establecido por los propios liberales del gobierno saliente de George Bush desmiente uno de los tendencias que Ohmae considera la mas importante.

Por lo demás, su esperanza cifrada en el "cibercontinente" parece como una utopía de alcances muy peligrosos, como si el mundo podría convertirse en una"realidad virtual" de espaldas a la realidad física; tomar decisiones en base a esa utopía nos lleva, por ejemplo, al desplome de las acciones "tecnológicas" de la Bolsa norteamericana, o al abandono de la economía física, en medio de una grave crisis energética y de alimentos.

Su paradigma de los Estados región, de espaldas a los continentes, se parece mucho al viejo esquema colonial británico de organizar sólo las zonas portuarias de un continente, excluyendo el vasto interior continental y desconectándola de él, con fines del comercio internacional. Estos enclaves portuarios tenían vías de transporte pero sólo para trasportar las mercaderías a exportarse, sin relación con el desarrollo de la propia economía física del país. Este esquema de enclaves fue totalmente rebasado por la realidad, $\mathrm{y}$ por las expectativas de los pueblo - continente que se independizaron a mediados del siglo XX.

De hecho, muchas de las tendencias de Ohmae -- como la regionalización y la reducción progresiva de los estados nacionales - ya se están poniendo en marcha, a nivel internacional, como políticas de la globalización, pero por otro lado las mismas políticas globalizadoras están en discusión a raíz de la crisis financiera mundial, y se está planteando a una vuelta a las estrategias contrarias de re- forzamiento del estado nacional de Franklin Delano Roosevelt, pero mejoradas y puestas al día, en países como los Estados Unidos, Rusia, China e India.

\section{CONCLUSIONES}

- El mérito de Ohmae es indudable, por su empeño en caracterizar a la globalización, puntualizando en sus características más importantes y divulgando muchos aspectos cruciales. Muchas de estas características son reales en lo descriptivo, aún presentando omisiones cruciales.

- Sin embargo, los elementos han sido reunidos en base a supuestos o tendencias de raíz ideológica, lo que hace que el conjunto pierda su carácter científico. Esto se demuestra en que las predicciones hechas en base a estos supuestos o tendencias, en especial en lo económico, han sido negadas por la realidad.

- La mayoría de la predicciones formuladas por Ohmae en lo económico en 2005, han sido negadas por la realidad de las crisis financiera norteamericana de 2008, y su reto al sistema del dólar,

- Muchas de las tendencias en lo político de Ohmae -- como la regionalización y el desmantelamiento de los estados nacionales - ya se están poniendo en marcha, a nivel internacional, como políticas de la globalización, pero estas tendencias políticos sufren un reto en lo económico, a raíz de la actual crisis financiera mundial, en la cual se está planteando a una vuelta a las estrategias clásicas de estado nación (que Ohmae rechaza) de Franklin Delano Roosevelt, pero mejoradas y puestas al día.

- Los factores que Ohmae deja de lado, como la geopolítica y el narcotráfico, pero también la irrupción de de estados nacio- 
nales de pueblos- continente como China, India y Rusia están tomando fuerza, en algunos casos a favor y en otros contrapuestos de las tendencias esbozadas.

- En el concepto del "Fin del Estado nación”, cuya conclusión sería su reemplazo en lo político por una forma de imperio global, Ohmae plantea una perspectiva mucho mas "aceptable" en la forma de los Estados región, pero con características utópicas, dado que, en la mayoría de los casos mencionadas, las experiencias regionales exitosas han tenido como apoyo al Estado nación, o, en otro caso, a alguna situación excepcional del mercado que no podría generalizarse. Ohmae también implica que, así como hay Estados región, el resto del territorio mundial pasará a la condición de "terra incógnita" o estados fallidos.

- En el caso del Perú, los potenciales Estados -región, ubicados en la costa, no cuentan con los todos los atributos señalados, en especial, infraestructura y educación científica. Adicionalmente, enfrentan las crisis de seguridad causada por las regiones - inviables- según el esquema de Ohmae -- dominadas por el narcotráfico y la violencia.

- Si bien Ohmae nos muestra muchas características de la globalización reales, las combina con una esperanza utópica en que el "cibercontinente" reemplazará en algún momento a los continentes reales, llenos de recursos y poblaciones pensantes y voluntariosas. Muchos de los pronósticos que Ohmae extrae de las tendencias esbozadas constituyen utopías ideologizadas, $y$ han sido negados por los hechos.
- El escenario mostrado por Ohmae de la globalización no tienen posibilidades de perdurar en su forma actual, debido a que la plataforma fundamental, el dólar, enfrenta una crisis existencial, cuya solución pasa por fortalecer el Estado nación, al contrario de lo postulado por el autor. Ohmae refleja indudablemente las disposiciones de las elites mundiales, pero no es sensato considerar como indudable que estas políticas utópicas tienen posibilidad de perdurar en el tiempo, como ya se están cuestionando por la crisis financiera internacional.

\section{REFERENCIAS BIBLIOGRÁFICAS}

1. Ohmae, Kenichi: "El próximo escenario global. Retos y Oportunidades en nuestro mundo sin fronteras -Wharton School Publishing. Segunda Edición, Madrid, Grupo Editorial Norma, 2008. . (1997). El fin del Estado-Nación. El ascenso de las Economías Regionales. Santiago de Chile: Andrés Bello. . (2004) La mente del Estratega. Mc Graw Hill interamericana de España. Madrid.

2. Brzezisnky, Zbigniew. (2003). El Gran Tablero Mundial. La Supremacía Estadounidense y sus Imperativos Geoestratégicos. Tercera Edición Buenos Aires: Ediciones Paidós, Ibéricas, S.A.

3. Drucker, Peter. (2003). La Sociedad que viene, editorial Empresa Activa, Madrid, 2003.

4. Leader Summaries. Resumen Autorizado de The Next Global Stage. The Challenges and Opportunities in our Borderless World. 2005 